\title{
DETERMINACIÓN DE ESPECTROS DE RESPUESTA CONSIDERANDO DAÑO ACUMULADO E INTERACCIÓN SUELO-ESTRUCTURA
}

\author{
Ali Rodríguez ${ }^{(1)}$, Edén Bojórquez Mora ${ }^{(2)}$, Alfredo Reyes-Salazar ${ }^{(2)}$, \\ Javier Avilés ${ }^{(3)}$ y Sonia E. Ruiz Gómez ${ }^{(4)}$
}

\begin{abstract}
RESUMEN
En este trabajo se evaluó en primer lugar la ductilidad cíclica en sistemas de un grado de libertad con comportamiento no lineal que representan estructuras sismo-resistentes considerando el efecto de las demandas acumuladas de deformación plástica e interacción suelo-estructura (ISE). Adicionalmente, se trazaron espectros de respuesta de ductilidad máxima y cíclica constante considerando ISE. Para alcanzar los objetivos del presente estudio, varios sistemas simplificados se sometieron a movimientos sísmicos registrados en sitios de suelo blando de la Ciudad de México, donde se ha observado efecto importante de ISE y daño acumulado. Se demostró que las demandas acumuladas de deformación plástica pueden tener influencia considerable en el comportamiento sísmico reduciendo la capacidad de ductilidad de las edificaciones, y por otro lado incrementando las ordenadas espectrales de manera importante. Finalmente, el impacto de la ISE depende principalmente de la flexibilidad del suelo, y puede tener efectos benéficos tanto como perjudiciales dependiendo de la razón entre el periodo del sistema y del suelo, incrementando o reduciendo la respuesta de una estructura.
\end{abstract}

Palabras clave: ductilidad cíclica; daño acumulado; interacción suelo-estructura

\section{DETERMINATION OF RESPONSE SPECTRA CONSIDERING CUMULATIVE DAMAGE AND SOIL STRUCTURE-INTERACTION}

\begin{abstract}
In this study, the cyclic ductility of single degree of freedom systems with non-linear behavior representing earthquake-resistant structures was estimated considering the effect of cumulative plastic deformation demands and soil-structure interaction (SSI). Additionally, earthquake response spectra in terms of peak and cyclic constant ductility taking into account SSI were computed. To achieve the aims of the present study, several simplified systems were subjected to earthquake ground motions recorded at soft soils sites of Mexico City where a significant effect of SSI and cumulative damage have been observed. It was shown that cumulative plastic deformation
\end{abstract}

Recibido el 31 de agosto de 2016, Revisado el 27 de junio de 2017, Aprobado el 28 de junio de 2017. Se aceptarán comentarios y/o discusiones hasta cinco meses después de su publicación.

(1) Maestría de la Facultad de Ingeniería, Universidad Autónoma de Sinaloa, Culiacán, Sinaloa, México, C.P. 80040. Actualmente Estudiante de Doctorado en Instituto de Ingeniería, UNAM, ali adio@ hotmail.com

(2) Facultad de Ingeniería, Universidad Autónoma de Sinaloa, Calzada de las Américas y Boulevard Universitarios S/N, Ciudad Universitaria, Culiacán, Sinaloa, México, C.P. 80040. eden@uas.edu.mx eden_bmseg@hotmail.com, reyes@uas.edu.mx

(3) Instituto Mexicano de Tecnología del Agua, Paseo Cuauhnahuac 8533, Jiutepec 62550, Morelos, México. javiles@tlaloc.imta.mx

(4) Instituto de Ingeniería, Universidad Nacional Autónoma de México, Ciudad Universitaria, Coyoacán 04510, México. sruizg@iingen.unam.mx 
demands have an important influence on the seismic behavior reducing the ductility capacity of structures and by the other hand increasing the spectral ordinates considerably. Finally, the impact of SSI depends mainly on the soil flexibility and could have beneficial or detrimental effects depending on the ratio of system and ground period, increasing or decreasing the response of a structure.

Keywords: cyclic ductility; cumulative damage; soil-structure interaction

\section{INTRODUCCIÓN}

Las metodologías actuales para el diseño sísmico de estructuras admiten la posibilidad de que durante excitaciones sísmicas severas ocurra un comportamiento plástico en edificaciones. Por ello, una estructura que se somete a varios ciclos de comportamiento plástico tendrá un efecto acumulado en términos de degradación y daño por fatiga, subsecuentemente la capacidad de deformación de la estructura se reduce. Aunque la deformación máxima y ductilidad son parámetros utilizados para considerar el comportamiento no lineal de una estructura, se ha reconocido que el daño estructural producto de excitaciones símicas no depende únicamente de estos parámetros, por consiguiente, el daño acumulado resultado de numerosos ciclos plásticos debería tomarse en consideración (Fajfar, 1992). Para tomar en cuenta la acumulación de las demandas plásticas en estructuras sismo-resistentes se han desarrollado diversos indicadores o índices de daño (Park y Ang, 1985; Terán y Jirsa, 2005; Rodríguez y Padilla, 2008; Bojórquez et al., 2009 y Bojórquez et al. 2010).

Por otra parte, es fundamental reconocer que el comportamiento sísmico no se encuentra influenciado únicamente por la respuesta de la superestructura, también por la respuesta de la cimentación y el suelo circundante. Para evaluar la respuesta sísmica de una estructura usualmente se supone que su base se encuentra empotrada, restringida contra traslación y rotación, esta suposición puede ser razonable para estructuras desplantadas en suelos relativamente rígidos; sin embargo, es necesario proceder con precaución cuando se analiza una estructura que se encuentra sobre un suelo flexible. Para sistemas elásticos, es conocido que la interacción suelo-estructura (ISE) alarga el periodo natural y provoca un cambio en el amortiguamiento modal para el modo fundamental de vibración (Avilés y Suarez, 2002). Dado que la mayoría de los códigos de diseño sísmico se basan en el uso de espectros de diseño, el incremento en el periodo de vibración y razón de amortiguamiento de una estructura permite suponer que la ISE disminuye la respuesta sísmica en algunos casos. Actualmente, para considerar la ISE, se usa un oscilador lineal de remplazo representado por el periodo y amortiguamiento efectivo del sistema (Jennings y Bielak, 1973; Veletsos y Meek, 1974 y Stewart et al. 2003). Sin embargo, esta aproximación puede que no sea aplicable al análisis de daño, donde la demanda máxima plástica y las demandas acumuladas de deformación plástica son cantidades de respuesta no lineal de gran interés (Avilés y Pérez-Rocha, 2007).

El objetivo de este estudio es evaluar la ductilidad cíclica en sistemas de un grado de libertad con comportamiento no lineal que representen estructuras sismo-resistentes, bajo consideración explicita del efecto de las demandas acumuladas de deformación plástica e ISE. Para ello, varios sistemas simplificados se someten a movimientos sísmicos registrados en sitios de suelo blando de la Ciudad de México, donde se ha observado el efecto importante de la ISE y daño acumulado.

\section{DAÑO ACUMULADO}

Para considerar la acumulación de las demandas plásticas en estructuras sismo-resistentes se han desarrollado diversos índices de daño. El objetivo de dichos índices es cuantificar el nivel de degradación 
o daño en un sistema considerando las propiedades de los materiales y las características de las acciones aplicadas (Arroyo y Ordaz, 2007). La evaluación del daño acumulado en estructuras sismo-resistentes puede abordarse desde diferentes perspectivas, algunas metodologías de daño resultan de la combinación lineal de las demandas de energía histerética normalizada y del desplazamiento máximo (Park y Ang 1985, Bozorgnia y Bertero 2001); de la energía histerética normalizada respecto a la máxima demanda elástica (Rodríguez 1997); o de la consideración explícita del número y amplitud de los ciclos de comportamiento plástico a través del índice de acumulación lineal del daño (Krawinkler y Zohrei 1983).

El impacto del uso de un índice de daño u otro, puede determinarse mediante la comparación de los resultados obtenidos a partir de su aplicación. Algunos investigadores estimaron que las demandas de resistencia derivadas del uso del índice de daño de Park y Ang (1985) son semejantes a las obtenidas de otros índices de daño planteados a partir de simplificaciones y suposiciones diferentes (Cosenza et al. 1993 y Terán y Jirsa, 2005). Por tal motivo, en el presente trabajo se utiliza el índice de Park y Ang (1985) para representar el daño estructural en osciladores no lineales.

\section{Índice de daño de Park y Ang}

Este índice de daño evalúa el nivel de daño estructural en elementos y estructuras de concreto reforzado sujetas a cargas cíclicas. Es uno de los más conocidos y usados debido a que existen numerosos estudios experimentales que lo sustentan, puede estimarse a partir de la combinación lineal de las demandas máximas y acumuladas de deformación plástica:

$$
I_{D P A}=\frac{\mu_{m}}{\mu_{u}}+\beta \frac{E_{H}}{F_{y} \delta_{y} \mu_{u}}
$$

donde $\mu_{m}$ es la ductilidad máxima que desarrolla la estructura cuando se sujeta a la acción de un movimiento sísmico, $\mu_{u}$ es la ductilidad última que puede alcanzar un sistema cuando está sujeto a un estado de deformación monótonamente creciente, $\beta$ es un parámetro que depende de las características estructurales, $E_{H}$ es la demanda de energía histerética, $F_{y}$ y $\delta_{y}$ son la fuerza y desplazamiento de fluencia, respectivamente.

En este estudio se utiliza $\beta$ de 0.15 , que corresponde a estructuras con un detallado sísmico adecuado de acuerdo a Cosenza et al. 1993. Teóricamente, cuando la energía disipada es nula el elemento o estructura se encuentra en el rango elástico, por lo cual no debería de existir daño y el $I_{D P A}$ debería ser igual a cero, mientras que un valor igual a la unidad indica la falla del sistema.

Peter Fajfar (1992) plantea que una vez que una estructura alcanza su rango de comportamiento plástico, su capacidad para disipar energía se reduce debido al daño acumulado causado por los ciclos de carga y descarga, en consecuencia una ductilidad equivalente debería usarse en lugar de la ductilidad monotónica creciente. A partir del caso de falla donde el $I_{D P A}=1$, la ductilidad equivalente o máxima permisible que un sistema puede desarrollar se obtiene de la siguiente manera (Fajfar, 1992):

$$
\begin{aligned}
& \mu_{m}=\frac{\sqrt{1+4 \beta \gamma^{2} \mu_{u}}}{2 \beta \gamma^{2}}-1 \\
& \gamma=\frac{\sqrt{E_{H} / m}}{\omega \delta_{m}}
\end{aligned}
$$


La ecuación 2 sugiere que la reducción de la ductilidad está controlada por el parámetro $\gamma$. Este parámetro adimensional, definido por la ecuación 3, depende de la frecuencia natural de la estructura $\omega$ y de la razón entre la raíz cuadrada de la energía histerética disipada por unidad de masa $m$ y el desplazamiento máximo $\delta_{m}$ (Fajfar, 1992).

\section{INTERACCIÓN SUELO-ESTRUCTURA}

La gran mayoría del análisis estructural se realiza bajo la suposición que la base de una estructura se encuentra empotrada, lo cual es aceptable para estructuras sobre suelos relativamente rígidos. Sin embargo, para estructuras desplantadas sobre un suelo flexible, la respuesta sísmica puede variar debido a dos efectos principales. Primeramente, modificación del movimiento de campo libre en la base de la estructura, segundo, un incremento en el periodo de vibración debido a la flexibilidad del suelo, y generalmente un aumento en el amortiguamiento generado por el comportamiento histerético y radiación de onda en el suelo. El primero se conoce como interacción cinemática y el segundo como interacción inercial, y todo el proceso es mejor conocido como interacción suelo-estructura (Wolf, 1985, Arefi, 2008 y Fernández et al., 2012).

Por tal motivo, la necesidad de comprender adecuadamente los efectos de ISE para incorporarlos al análisis estructural de una manera práctica y averiguar en cuales casos son benéficos o perjudiciales sobre la respuesta sísmica. Avilés y Pérez-Rocha (2004) analizaron numéricamente un modelo simplificado suelo-estructura, basados en estos análisis propusieron un oscilador equivalente de base-rígida con la capacidad de incursionar en el rango plástico, el cual se describe a continuación.

\section{Modelo simplificado suelo-estructura}

El sistema suelo-estructura consistió en una estructura elasto-plástica de un nivel soportada por una cimentación rígida, la cual se encuentra insertada en un estrato homogéneo y viscoelástico de base rígida, como se muestra en la figura 1.

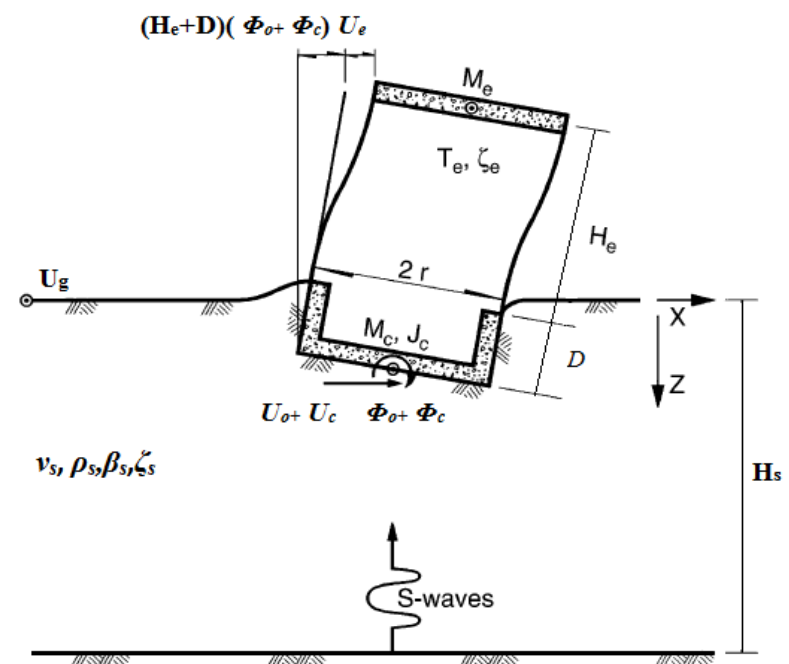

Figura 1. Estructura elasto-plástica insertada en un estrato con base rígida (Avilés y Pérez-Rocha, 2004)

La estructura se caracteriza por su altura $H_{e}$ y masa $M_{e}$; el periodo natural y la razón de amortiguamiento para la condición de base-rígida está dado por $T_{e} \mathrm{y} \zeta_{e}$, respectivamente. La cimentación se asume como circular de radio $r$, con una profundidad de empotramiento $D$, masa $M_{c}$ y momento de inercia de masa $J_{c}$ respecto a su eje centroidal de rotación, cabe mencionar que el modelo no considera la 
no-linealidad de la cimentación. El estrato de espesor $H_{S}$, está caracterizado por la razón de Poisson $v_{\mathrm{S}}$, densidad de masa $\rho_{S}$, velocidad de onda de corte $\beta_{S}$ y razón de amortiguamiento histerético $\zeta_{s}$.

El desplazamiento horizontal en la superficie es generado por el movimiento de campo libre $U_{g}$. La componente torsional no se genera. Los tres grados de libertad del sistema son el desplazamiento relativo de la estructura $U_{e}$, el desplazamiento de la cimentación $U c$ relativo al movimiento de entrada $U_{o}$ y la rotación de la cimentación $\Phi_{c}$ relativo al movimiento de entrada rotacional $\Phi_{o}$.

Para los resultados presentados, se asumieron los siguientes parámetros representativos (Avilés y Pérez-Rocha, 2004): $M c / M e=0.25, J_{c} / M_{e}\left(H_{e}+D\right)^{2}=0.05, M_{e} / \rho_{S} \pi^{2} H_{e}=0.15, \zeta_{e}=\zeta_{s}=0.05$ y vs=0.45. Estos valores pretenden ser aproximados para edificaciones típicas y para condiciones de suelo flexible.

\section{Oscilador equivalente no-lineal}

Avilés y Pérez-Rocha (2003), basados en los análisis realizados al modelo anterior, demostraron que una estructura de base-flexible puede ser remplazada apropiadamente por un oscilador equivalente de base-rígida, el cual está caracterizado por el periodo y el amortiguamiento efectivo del sistema. Estos últimos se obtienen conforme a las ecuaciones 4 y 5.

$\tilde{T}_{e}=\sqrt{T_{e}^{2}+T_{h}^{2}+T_{r}^{2}}$

$$
\tilde{\zeta}_{e}=\frac{1}{Q_{h}+\left(H_{e} / r+D / r\right) Q_{r}} \times\left(\zeta_{e} \frac{T^{3}}{\tilde{T^{3}}}+\frac{\zeta_{h}}{1+2 \zeta_{h}{ }^{2}} \cdot \frac{T_{h}{ }^{2}}{T^{2}}+\frac{\zeta_{r}}{1+2 \zeta_{r}{ }^{2}} \cdot \frac{T_{r}{ }^{2}}{T^{2}}\right)
$$

donde $T_{h}=2 \pi \sqrt{M_{e} / K_{h}}$ y $T_{r}=2 \pi \sqrt{M_{e}\left(H_{e}+D\right)^{2} / K_{h}}$ son los periodos que tendría la estructura si fuera infinitamente rígida y su base solo pudiera trasladarse o rotar, los parámetros $K_{h}$ y $K_{r}$ representan las

rigideces de la cimentación. Mientras $\zeta_{h}=\pi C_{h} / \tilde{T}_{e} K_{h}$ y $\zeta_{r}=\pi C_{r} / \tilde{T}_{e} K_{r}$ son los coeficientes de amortiguamiento del suelo en los modos de traslación y rotación, los parámetros $C_{h}$ y $C_{r}$ representan los amortiguamientos de la cimentación, por último $Q_{h}$ y $Q_{r}$ son las funciones de transferencia en los modos de translación y rotación (NTCDS, 2004). Estas cantidades representan las funciones de impedancia dependientes de la frecuencia.

Finalmente, con el propósito que el oscilador equivalente tenga la misma resistencia a la fluencia y capacidad de deformación que una estructura real de base-flexible, se requiere un factor de ductilidad equivalente o ductilidad efectiva, la cual puede expresarse como (Avilés y Pérez-Rocha, 2004):

$$
\tilde{\mu_{e}}=1+\left(\mu_{u}-1\right) \frac{T_{e}^{2}}{\tilde{T e^{2}}}
$$

Los desplazamientos involucrados en el oscilador equivalente son el desplazamiento de la estructura Ue y el desplazamiento de cuerpo rígido $\mathrm{Uc}+(\mathrm{He}+\mathrm{D}) \Phi \mathrm{c}$ inducido por la translación y rotación de la cimentación. Este último movimiento es responsable de la reducción de ductilidad del sistema. 


\section{Funciones de impedancia}

La tarea más compleja en la determinación del periodo y amortiguamiento efectivo es el cálculo de las funciones de impedancia. Existen diferentes métodos disponibles para calcular las funciones de impedancia, los cuales se basan en técnicas de transformación integral, método de elemento finito, método de elemento de frontera y algunos otros en métodos híbridos (Gazetas, 1991). Lo anterior complica los cálculos; además, demanda un tiempo y esfuerzo computacional significativo. Sin embargo, para propósitos ingenieriles existen fórmulas aproximadas. En esta investigación, las fórmulas utilizadas para calcular los coeficientes dinámicos de las funciones de impedancia y considerar el movimiento de entrada de la cimentación se encuentran en la tabla A.2 del Apéndice A, perteneciente a la versión 2004 de las Normas Técnicas Complementarias para Diseño por Sismo (NTCDS).

\section{METODOLOGÍA}

Para la evaluación de la ductilidad equivalente y la determinación de espectros de respuesta, se utilizó un total de 68 movimientos sísmicos registrados en las zonas IIIa, IIIb, IIIc y IIId de la Ciudad de México durante eventos sísmicos de subducción. Las tablas A1-A4 incluidas en el Apéndice A de este artículo clasifican los registros sísmicos conforme a lo establecido por el cuerpo principal de las NTCDS; además, resumen algunas de sus características más relevantes. Los grupos de registros sísmicos se tomaron de un estudio realizado por Villa-Velázquez y Ruiz (2001), y se escalaron para ser congruentes con el nivel de aceleraciones que establecen las NTCDS para cada zona.

La ductilidad equivalente se evaluó primeramente considerando daño acumulado; posteriormente, tomando en cuenta ISE y daño acumulado simultáneamente. Se siguió un proceso similar para el cálculo de los espectros de respuesta. El índice de Park y Ang y el oscilador equivalente de base-fija se utilizaron para evaluar los efectos de las deformaciones plásticas acumuladas e ISE, respectivamente. Se emplearon sistemas de un grado de libertad con periodos de vibración que van de 0.1 a 5 segundos y amortiguamiento crítico de $5 \%$. Se asumió un comportamiento elasto-plástico perfecto; y ductilidades últimas de 2, 4 y 6, que representan sistemas con capacidades de deformación baja, alta y muy alta, respectivamente. Los sistemas con ductilidad igual a 6 , se utilizaron para aumentar el conocimiento sobre el comportamiento de sistemas no lineales que puedan desarrollar ductilidades muy altas, como el caso de ciertas estructuras de acero.

Por otra parte, la evaluación de los efectos de ISE se llevó a través de un análisis paramétrico, cuya finalidad fue cubrir un intervalo amplio de interés, por tal motivo se consideraron los siguientes parámetros adimensionales: relación de esbeltez de la estructura, $H_{e} / r$; relación de empotramiento de la cimentación, $D / r$; profundidad normalizada del estrato flexible, $H_{s} / r$; y la rigidez relativa entre la estructura y el suelo, $H_{e} / T_{e} \beta_{s}$. Estos parámetros tienen una gran correlación con los efectos de ISE acorde con algunos investigadores (Jennings y Bielak, 1973, Veletsos y Nair, 1974, Avilés y Suarez, 2002, Avilés y Pérez-Rocha, 2004, y Avilés y Pérez-Rocha, 2007).

A dichos parámetros se les asignaron los siguientes valores; $H_{e} / r=2,3$ y 5 que representaron estructuras de baja a gran altura, $D / r=0$ (soportadas-superficialmente), 0.5 y 1 (empotradasprofundamente) los cuales simularon el empotramiento de la cimentación y $H_{s} / r=2$ (superficial), 3 y 5 (profundo) para estratos que variaron de forma superficial a gran profundidad. Además, se asumió la relación empírica $H_{e} / T_{e} \approx 25 \mathrm{~m} / \mathrm{s}$ (Avilés y Pérez-Rocha, 2004). Note que para cualquier valor de $T_{e}$, el valor de $H_{e}$ se obtuvo de la razón constante $H_{e} / T_{e}$. Con esto, el valor de $r$ se determinó de la razón de esbeltez $H_{e} / r$, sucesivamente, el valor de $D$ se calculó de la razón de empotramiento $D / r$. Esto implica que la estructura cambio de altura como función del periodo y que las dimensiones de la cimentación variaron 
cuando la altura de la estructura cambio, como sucede con la mayoría de los edificios (Avilés y PérezRocha, 2004). Posteriormente, se determinaron las particularidades de los movimientos del terreno mediante una serie de espectros elásticos promedio. Cuyo propósito fue obtener el periodo dominante del suelo para las zonas investigadas, $T_{s}$, el cual se definió como el periodo donde ocurre la ordenada máxima dentro de un espectro elástico de pseudo-aceleración. La tabla 1 muestra el intervalo de periodos del suelo bajo consideración para cada zona.

Tabla 1. Intervalo de periodos considerado en cada zona sísmica de suelo blando.

\begin{tabular}{ccc}
\hline Zonas & Intervalo de periodos (s) & Periodo promedio (s) \\
\hline Zona IIIa & $1.1-1.5$ & 1.3 \\
\hline Zona IIIb & $1.8-2.2$ & 2.0 \\
\hline Zona IIIc & $2.8-3.2$ & 3.0 \\
\hline Zona IIId & $3.6-4.4$ & 4.0 \\
\hline
\end{tabular}

La figura 2 muestra los espectros elásticos promedio para las distintas zonas sísmicas investigadas, las ordenadas espectrales corresponden a la pseudo-aceleración, $S_{a}$, expresada como fracción de la aceleración de la gravedad, $g$. Mientras, el eje horizontal se refiere al periodo estructural, $T_{e}$. Los periodos del suelo obtenidos pretenden ser aproximados para cada zona. Dado que el periodo del suelo se encuentra relacionado con el daño estructural, servirá de referencia para análisis de resultados en esta investigación.
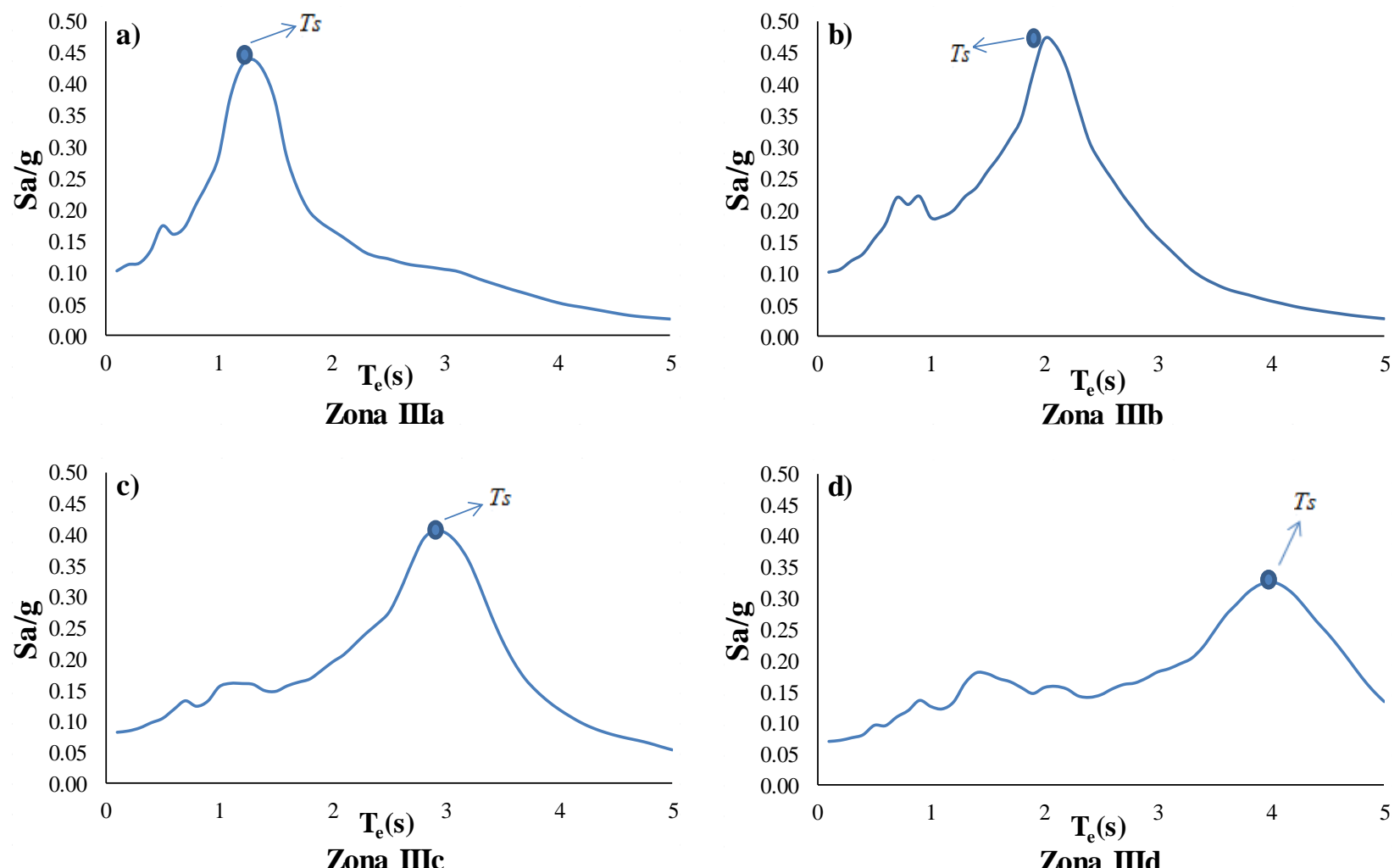

Figura 2. Espectros elásticos promedio de pseudo-aceleración correspondientes a distintas zonas de suelo blando de la Ciudad de México 


\section{DUCTILIDAD EQUIVALENTE CONSIDERANDO DAÑO ACUMULADO}

En esta sección se presentan los resultados obtenidos de la ductilidad equivalente como función del periodo estructural, cuya evaluación se llevó a cabo de acuerdo a la ductilidad máxima permisible que un sistema puede desarrollar en términos del índice de daño de Park y Ang (ver ecuación 2). La figura 3 muestra la ductilidad equivalente promedio para cada zona obtenida. En la figura, se observa que para estructuras con periodos cortos el daño producido por las demandas acumuladas de deformación plástica no tuvo gran repercusión. Por otro lado, para estructuras con periodos cercanos al periodo del suelo, existe una influencia significativa entre la relación del periodo estructural y el periodo del suelo, ya que se obtuvieron valores de ductilidad equivalente del $50 \%$ al $60 \%$ respecto a la ductilidad última, $\mu_{u}$. Para estructuras con periodos que se alejan del periodo dominante del suelo, la reducción de ductilidad depende en gran medida de $\mu_{u}$, debido a que se alcanzaron valores de ductilidad equivalente menores conforme $\mu_{u}$ se incrementó. Sin embargo, estas reducciones no son del mismo orden de las identificadas en la condición de resonancia. Para las zonas IIIc y IIId las tendencias observadas son las mismas, con la diferencia que incluso para periodos que se alejan del periodo del terreno, los valores de la ductilidad equivalente siguieron disminuyendo. Puede concluirse que los sistemas con periodos cortos son capaces de desarrollar una ductilidad mayor en comparación con los sistemas con periodo largo, como lo señalan Tapia y Tena (2014); además, cuanto mayor es el periodo del suelo, mayor es el rango de periodos estructurales afectado por las demandas acumuladas. Por ejemplo, en las zonas IIIc y IIId ocurren reducciones de ductilidad extremas para periodos estructurales mayores al periodo dominante del terreno.

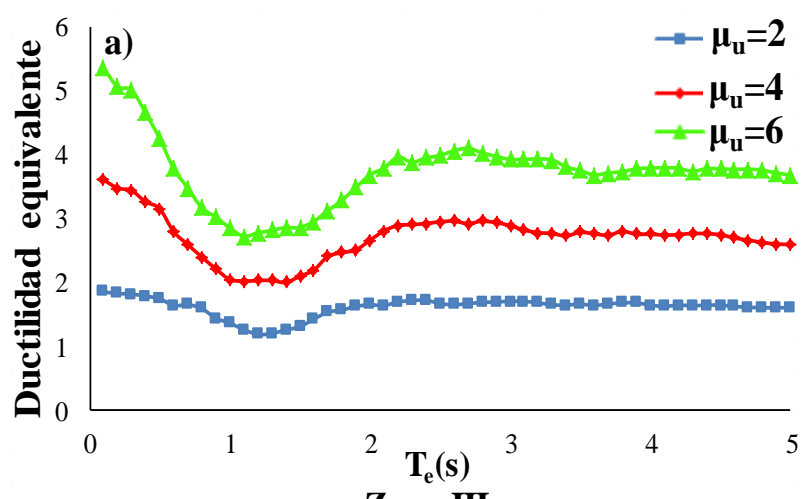

Zona IIIa

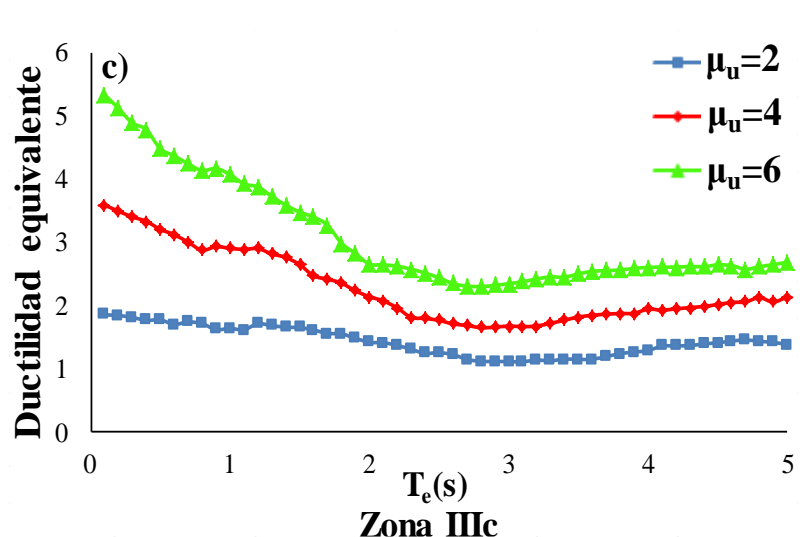

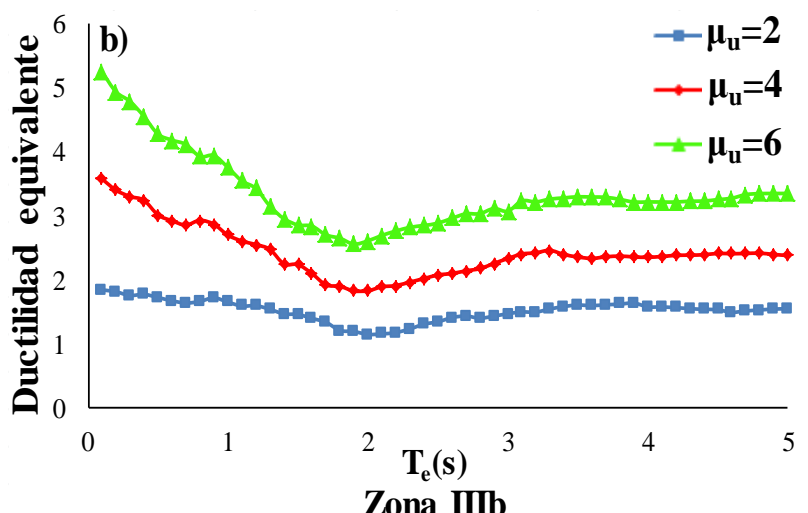

Zona IIIb

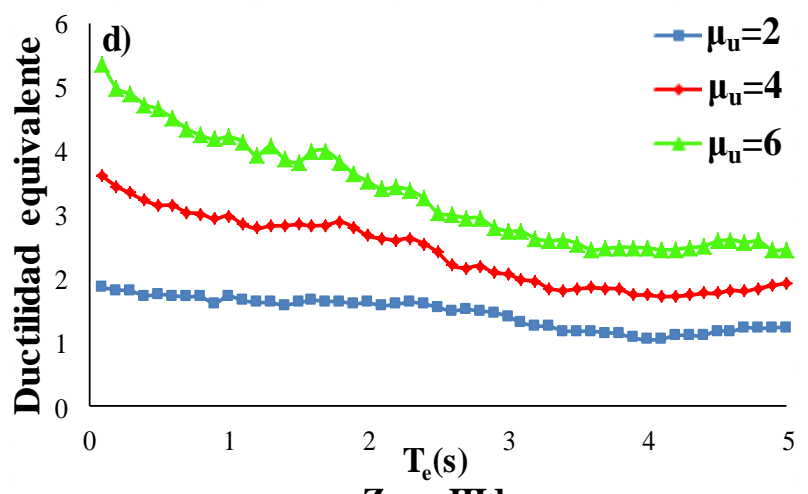

Zona IIId

Figura 3. Ductilidades equivalentes correspondientes a las zonas sísmicas de las Ciudad de México

Los resultados presentados en la figura anterior, se interpretan de manera más sencilla si el eje de las abscisas considera el periodo estructural normalizado por el periodo dominante del terreno. La figura 4 muestra que cuando el periodo estructural y el periodo dominante del terreno fueron similares, menor fue 
la capacidad de deformación de la estructura; además, para periodos estructurales que van de $0.75 T_{s}$ a $1.25 T_{s}$, la ductilidad se redujo aproximadamente $50 \%$ a $60 \%$. Es importante mencionar que para las zonas IIIa y IIIb, los valores de ductilidad equivalente se incrementaron conforme el periodo de la estructura se alejó del periodo del terreno, mientras tanto, las zonas IIIc y IIId, debido al alto contenido de energía en los movimientos del suelo, la ductilidad equivalente continuó disminuyendo en un intervalo de periodos mayor. Por otra parte, la Figura 5 muestra la ductilidad equivalente \pm una desviación estándar $\sigma$. Se observa que la dispersión en la estimación de la ductilidad equivalente es baja, por lo que puede concluirse que los registros seleccionados son adecuados ya que no existe incertidumbre considerable en la evaluación de la ductilidad equivalente.

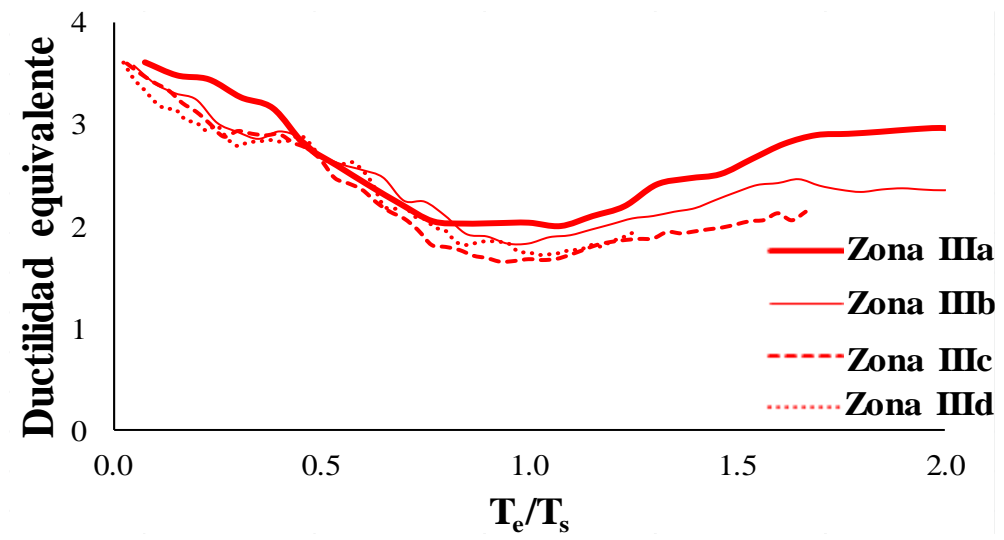

Figura 4. Ductilidades equivalentes correspondientes a las distintas zonas sísmicas de las Ciudad de México, $\mu=4$
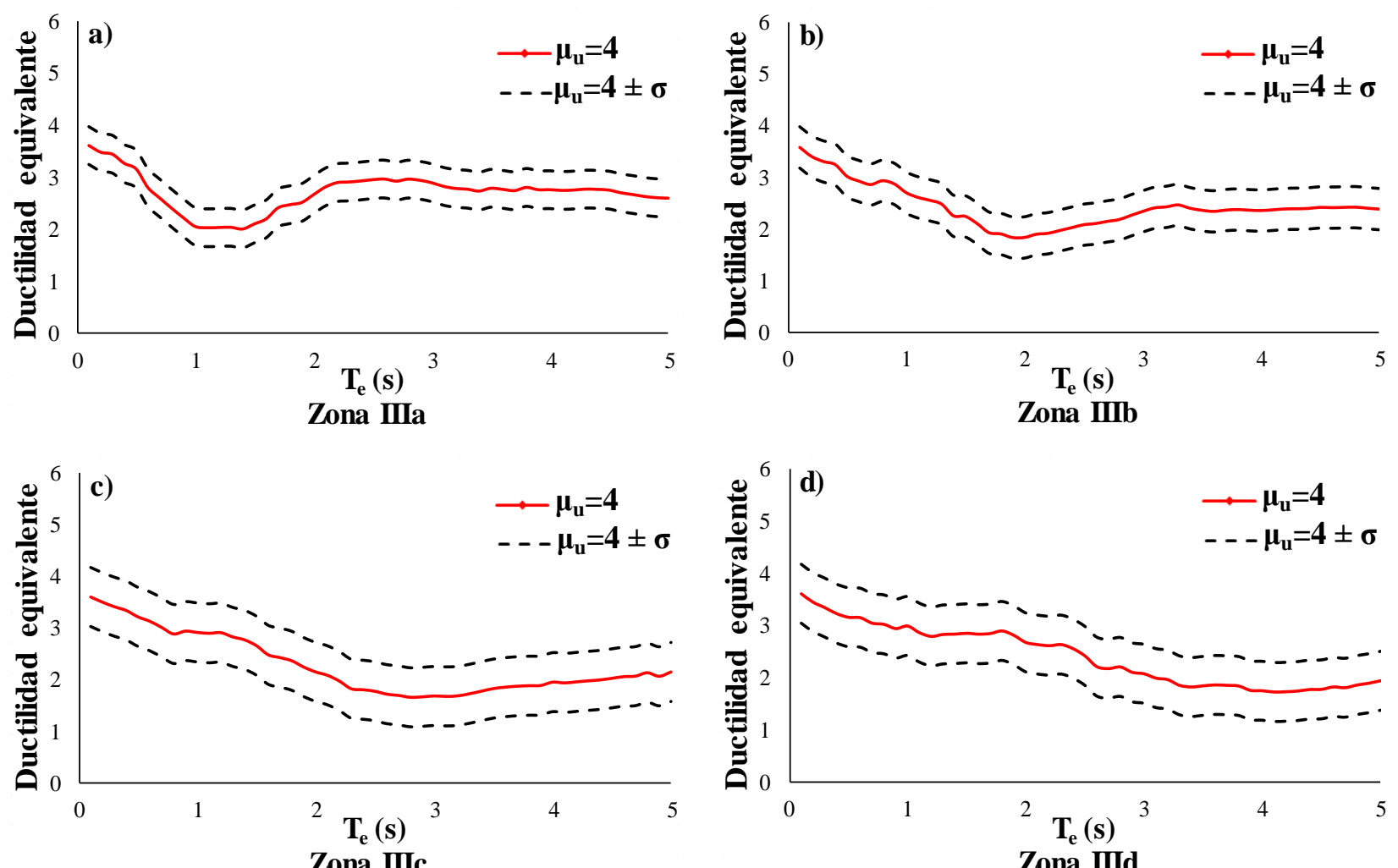

Figura 5. Ductilidades equivalentes correspondientes a las distintas zonas sísmicas de las Ciudad de México, $\mu=4 \pm \sigma$ 


\section{ESPECTROS DE RESPUESTA DE DUCTILIDAD CONSTANTE CONSIDERANDO DAÑO ACUMULADO}

Dado que los códigos de diseño sísmico se apoyan en el uso de espectros de pseudo-aceleración para estimar la resistencia lateral de una estructura, se determinaron espectros de respuesta promedio tomando en consideración el efecto del daño acumulado, y se analizó la influencia que las deformaciones plásticas tuvieron sobre la demanda de resistencia obtenida de un espectro de pseudo-aceleración.

La figura 6 muestra los espectros de respuesta promedio para cada una de las zonas sísmicas investigadas. El lado izquierdo de la figura muestra los espectros obtenidos sin contemplar el daño acumulado (Sin daño), mientras tanto, el lado derecho considerando explícitamente el efecto de las deformaciones plásticas (Con daño). Se observó que el efecto de las demandas plásticas acumuladas incrementa las ordenadas de resistencia en periodos estructurales próximos al periodo del terreno, el cual fue aproximadamente de $40 \%$ al $50 \%$ para todas las zonas, dichos porcentajes fueron similares a los obtenidos en la sección anterior. Además, mientras mayor fue el periodo dominante del suelo y el valor de ductilidad última, menor fue el periodo donde las máximas demandas de resistencia ocurrieron. Por ejemplo, para la zona IIIa con $T_{s}=1.3 \mathrm{~s}$, el aumento de resistencia más significativo ocurrió en $T_{e}=1.2,1.1 \mathrm{y}$ $0.9 \mathrm{~s}$ para $\mu_{u}=2,4$ y 6 , respectivamente. Lo mismo para la zona IIId con $T_{s}=4 \mathrm{~s}$, donde el incremento de resistencia más considerable sucedió en $T_{e}=3.7,3.0$ y $2.7 \mathrm{~s}$ para $\mu_{u}=2,4$ y 6 , respectivamente.

\section{DUCTILIDAD EQUIVALENTE CONSIDERANDO DAÑO ACUMULADO E ISE}

A continuación se evaluó la ductilidad equivalente considerando simultáneamente el efecto de las demandas acumuladas e ISE. Los resultados presentados corresponden únicamente a la zona IIIa por limitación de espacio. La figura 7 muestra la ductilidad equivalente promedio para la zona IIIa considerando daño acumulado, ISE y distintas velocidades de onda de corte, $\beta_{s}$. Las líneas punteadas cargadas a la derecha indican el periodo del suelo $T_{s}$, mientras que las líneas punteadas sobre la izquierda muestran el periodo estructural $T_{e}$, donde ocurrieron las mayores reducciones de ductilidad. Debe considerarse que, los valores sobre el eje de las abscisas corresponden a periodos de sistemas que se supusieron sobre base-rígida y no al periodo efectivo del sistema. Por ejemplo, para la velocidad de onda de corte $\beta_{s}=50 \mathrm{~m} / \mathrm{s}$, el periodo estructural donde ocurrió la mayor reducción de ductilidad fue $T_{e}=0.7 \mathrm{~s}$, lo cual es aproximadamente la mitad del valor del periodo del suelo. Esto indica que para $T_{e}=0.7 \mathrm{~s}$ el periodo efectivo debió ser aproximadamente $\widetilde{T}_{e}=1.3 \mathrm{~s}$; consecuente, coincidió con el periodo del suelo $T_{s}=1.3 \mathrm{~s}$. Por esta razón, las reducciones de ductilidad fueron tan significativas en dicho periodo. Además, para periodos estructurales cortos, la influencia de las demandas plásticas acumuladas en conjunto con los efectos de ISE causaron reducciones de ductilidad de $50 \%$ al $60 \%$ respecto a $\mu_{u}$, lo cual contrasta con los resultados obtenidos en secciones anteriores, donde se evaluó la ductilidad considerando exclusivamente daño acumulado, cuyo efecto no fue tan significante. Para $\beta_{s}=50 \mathrm{~m} / \mathrm{s}$, la mayor reducción de ductilidad fue aproximadamente de $25 \%$ a $55 \%$ respecto a $\mu_{u}$ y ocurrió en $T_{e}=0.7 \mathrm{~s}$. Los resultados mencionados difieren de aquellos obtenidos en secciones previas, donde los avalores de ductilidad fueron de $50 \%$ a $60 \%$ en relación a $\mu_{u}$ para estructuras próximas al periodo del terreno. La diferencia entre los resultados fue que aunado a la consideración de daño acumulado, también se incluyó la ISE. Por otra parte, para $\beta_{s}=180 \mathrm{~m} / \mathrm{s}$ y $T_{e}=1.2 \mathrm{~s}$, los valores de ductilidad equivalente conseguidos fueron entre $50 \%$ a $60 \%$ respecto a $\mu_{u}$, igual a aquellos presentados en secciones previas. La similitud se encuentra en que, para valores cercanos a $\beta_{s}=150 \mathrm{~m} / \mathrm{s}$, los efectos de ISE fueron bajos, por lo que el periodo y el amortiguamiento efectivo fueron similares al periodo y amortiguamiento de un sistema que se supone sobre base rígida. La discusión anterior recae en el hecho que los efectos de ISE están controlados principalmente por el parametro $H_{e} / T_{e} \beta_{s}$, el cual es uno de los parametros mas usuales para considerar la influencia de la ISE. Dado que se asumió la relación constante $H_{e} / T_{e} \approx 25 \mathrm{~m} / \mathrm{s}$, el parámetro $H_{e} / T_{e} \beta_{s}$, mide solamente la flexibilidad del suelo, y se incrementara para valores de $\beta_{s}$ menores. 

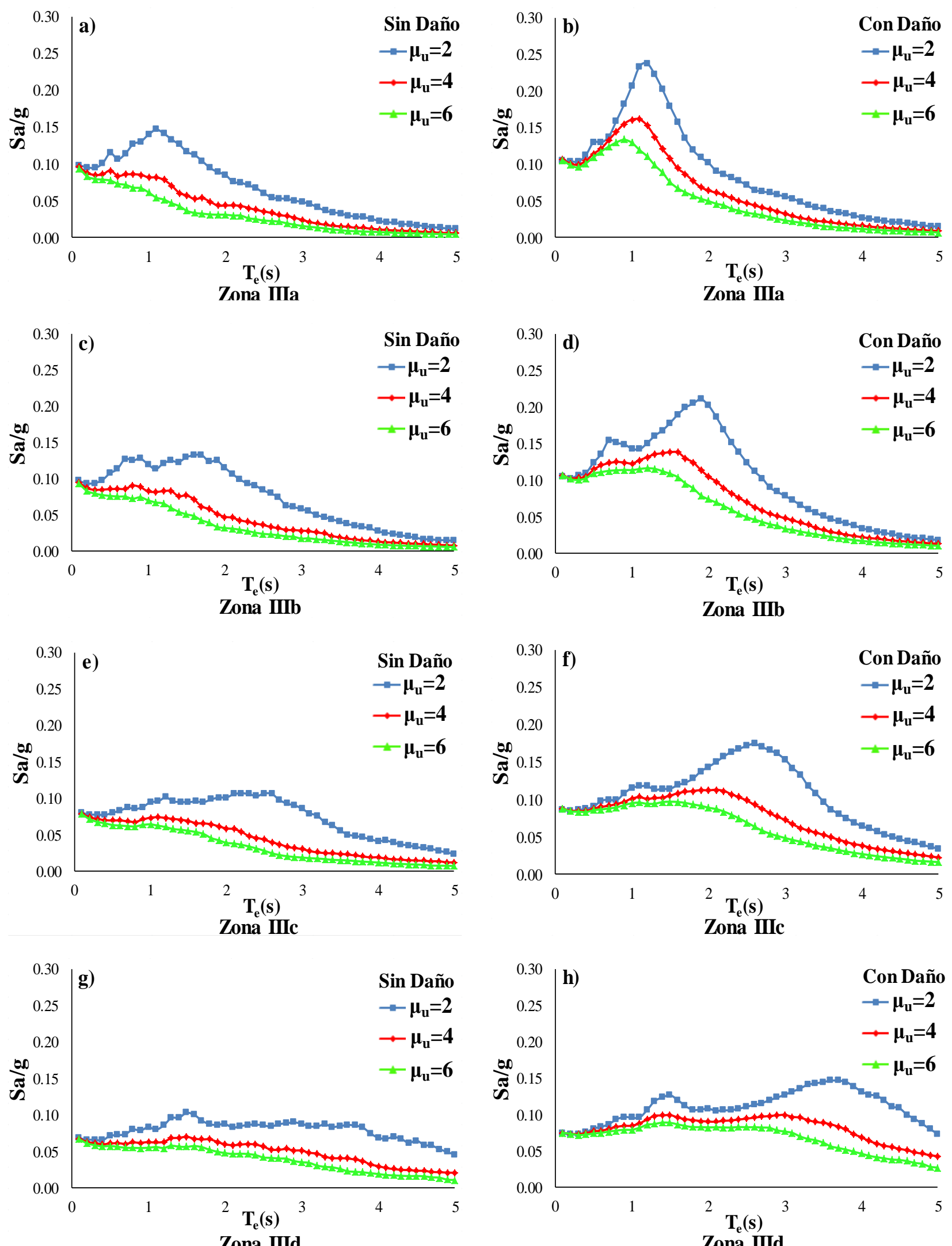

Figura 6. Espectros de respuesta promedio para sistemas elasto-plásticos; $\mu_{\mathrm{u}}=2$, 4 y 6 
Finalmente, mientras menor fue la velocidad de onda de corte, las reducciones de ductilidad fueron más notables, además, ocurrieron en un periodo estructural menor al periodo del sitio. Contrariamente, a medida que se aumentó el valor de la onda de corte, las mayores reducciones de ductilidad sucedieron en un periodo estructural similar al periodo dominante del suelo. Las líneas punteadas correspondientes a $T_{e} \mathrm{y}$ $T_{s}$ sirven como referencia.

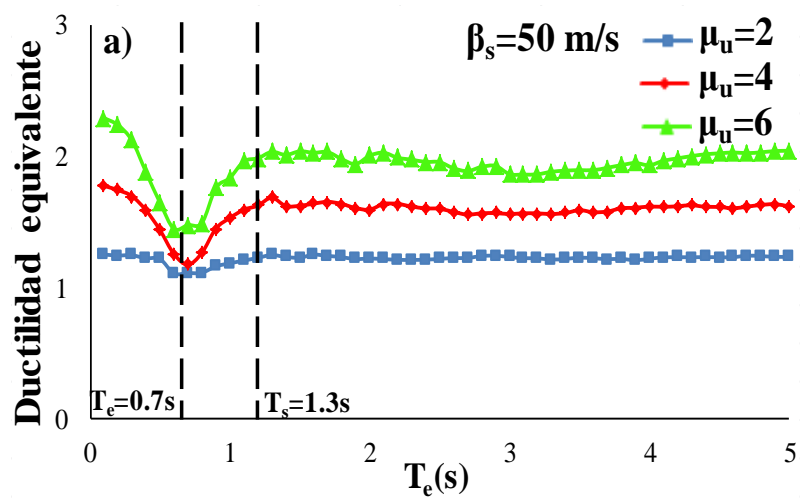

Zona IIIa

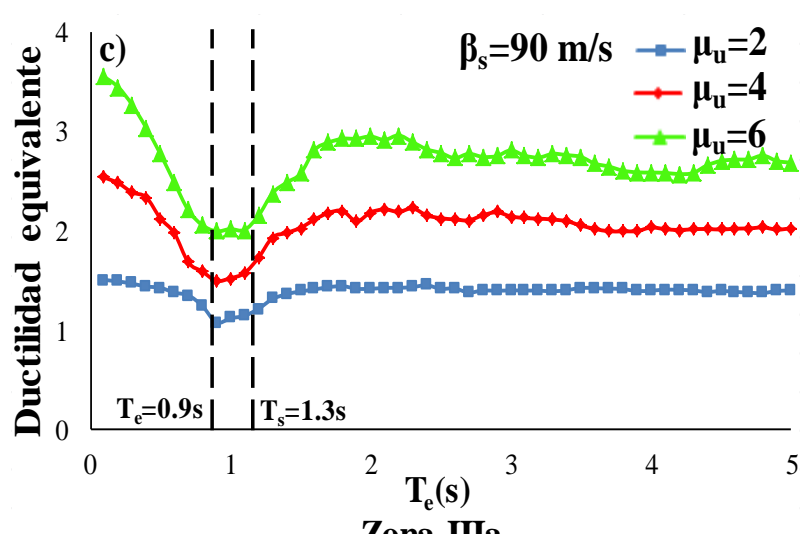

Zona IIIa

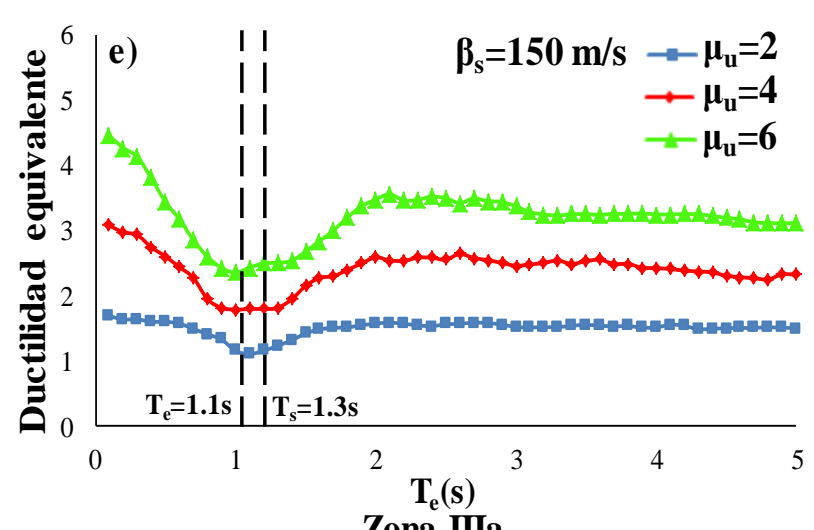

Figura 7. Ductilidad equivalente considerando ISE para distintas velocidades de ondas de corte $\beta \mathrm{s}$. Hs/r=3, $\mathrm{He} / \mathrm{r}=3$ y $\mathrm{D} / \mathrm{r}=0.5$
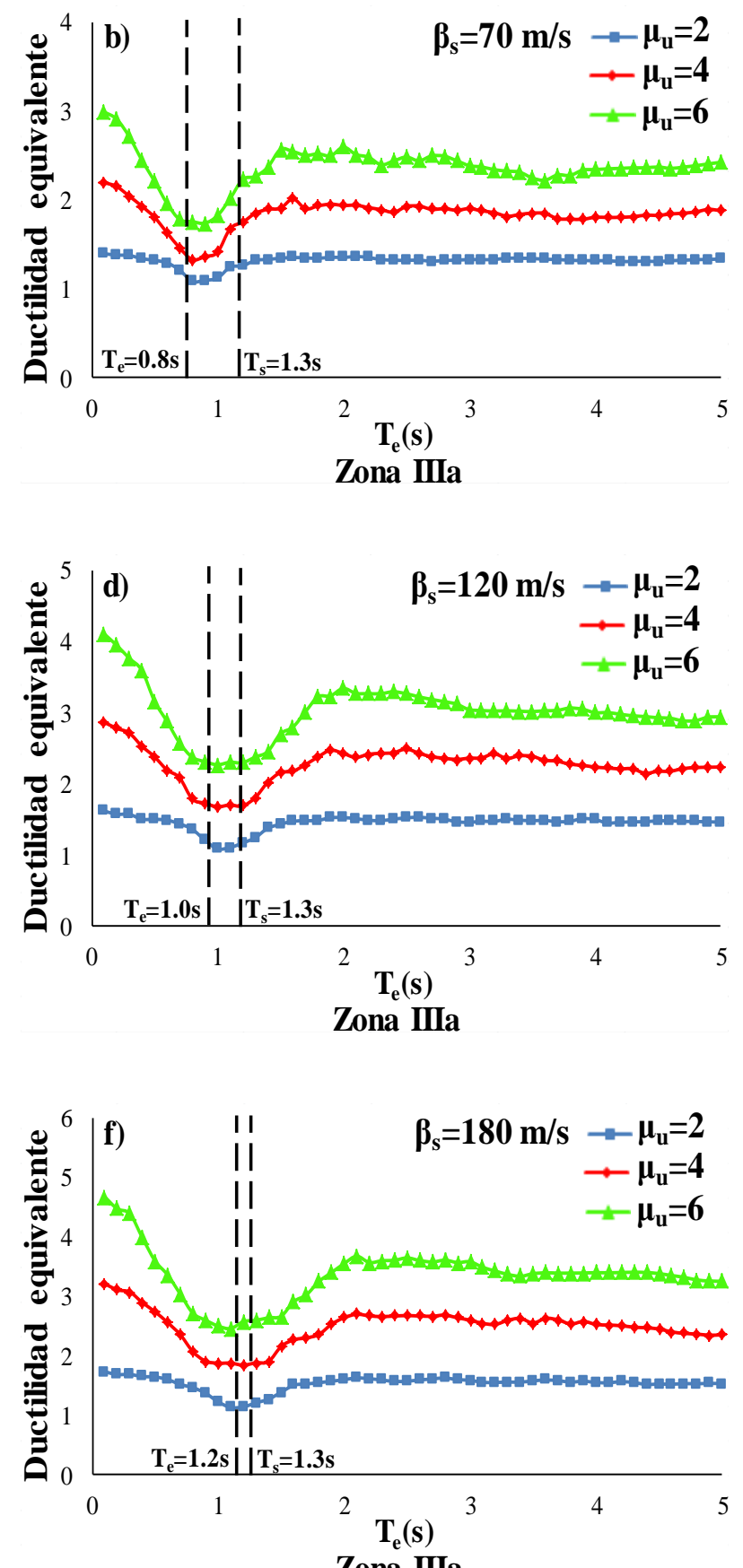

Zona IIIa

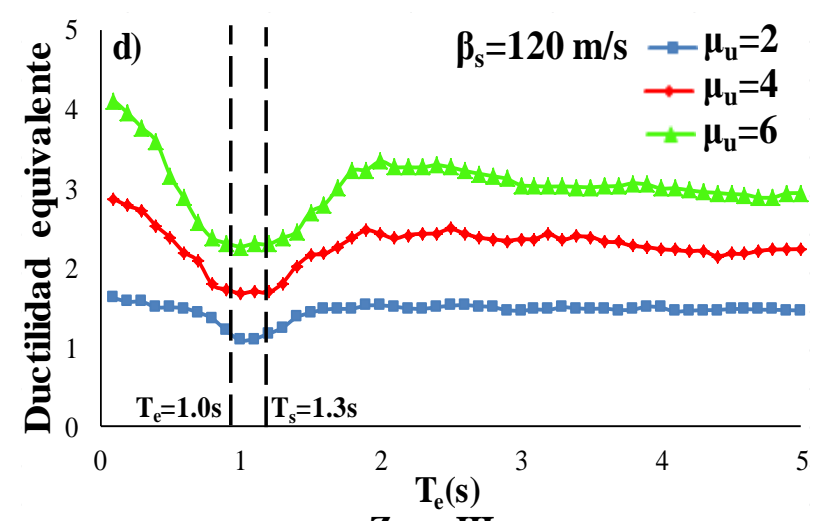

Zona IIIa

\section{Zona IIIa}


Las figuras 8 y 9 muestran la ductilidad equivalente promedio de las zonas IIIa y IIIb, para distintas velocidades de onda de corte y dos valores de ductilidad última. Se observó que mientras mayor fueron los valores del parámetro $H_{e} / T_{e} \beta_{s}$, mayor fue el incremento en el periodo del sistema. Considerando que lo anterior se obtuvo al evaluar $\beta_{s}=50,70$ y $90 \mathrm{~m} / \mathrm{s}$, por lo tanto, para estas cantidades, las máximas reducciones de ductilidad se produjeron en periodos estructurales mucho menores al periodo del terreno. Adicionalmente, para valores de $H_{e} / T_{e} \beta_{s}$ mayores a 0.15 , el periodo efectivo y el periodo estructural fueron similares $T_{e} \approx \widetilde{T}_{e}$. Tal como se ilustra, para $\beta_{s}=150 \mathrm{y} 180 \mathrm{~m} / \mathrm{s}$, la ductilidad equivalente fue semejante a la obtenida al considerar únicamente las demandas de deformación plástica. Por esta razón, a medida que se incrementó la velocidad de onda de corte, los efectos de ISE se tornaron poco significativos.

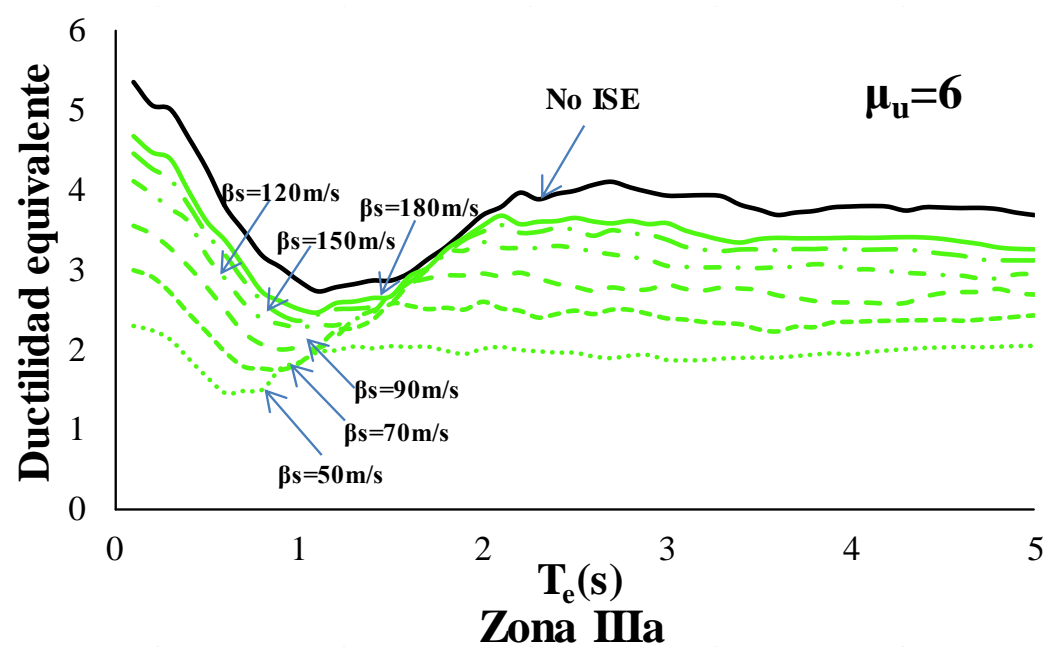

Figura 8. Ductilidad promedio considerando ISE para distintas ondas de corte $\beta \mathrm{s}$. $\mathrm{Hs} / \mathrm{r}=3, \mathrm{He} / \mathrm{r}=3 \mathrm{y}$ $\mathrm{D} / \mathrm{r}=0.5$ (Zona IIIa)

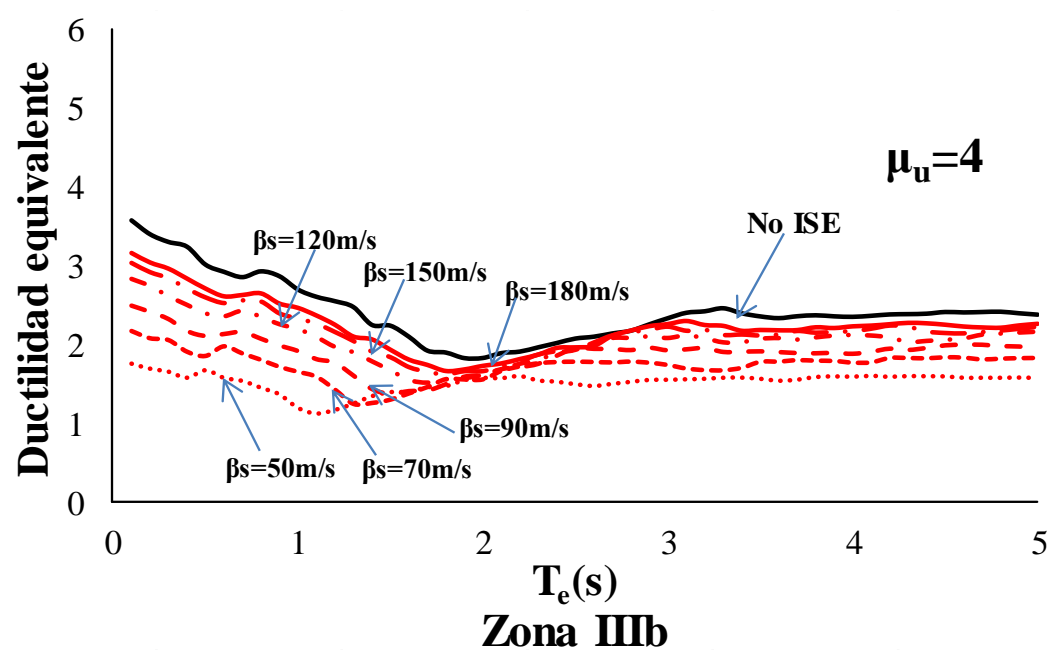

Figura 9. Ductilidad promedio considerando ISE para distintas ondas de corte $\beta \mathrm{s}$. Hs/r=3, He/r=3 y $\mathrm{D} / \mathrm{r}=0.5$ (Zona IIIb) 


\section{ESPECTROS DE RESPUESTA DE DUCTILIDAD CONSTANTE CONSIDERANDO DAÑO ACUMULADO E ISE}

La figura 10 presenta los espectros de respuesta promedio para $\beta_{s}=70 \mathrm{~m} / \mathrm{s}, \mathrm{H}_{\mathrm{s}} / \mathrm{r}=3, \mathrm{H}_{\mathrm{e}} / \mathrm{r}=3 \mathrm{y} \mathrm{D} / \mathrm{r}=0.5$, concernientes a cada una de las zonas sísmicas investigadas. Los espectros del lado izquierdo se obtuvieron al evaluar únicamente los efectos de ISE, mientras los espectros del lado derecho al considerar conjuntamente los efectos de las demandas plásticas acumuladas e ISE (ISE+Daño).

La diferencia entre los espectros que evalúan los efectos de ISE respecto aquellos que únicamente consideran daño acumulado, residió en que las mayores demandas de resistencia ocurrieron en periodos menores al periodo dominante del terreno. Aunque el efecto del daño acumulado no se incluyó en los espectros de la izquierda (ISE), existió un incremento en la resistencia producto de la ductilidad efectiva (ver ecuación 6), mientras que en los espectros de la derecha (ISE+Daño), la demanda de resistencia aumentó debido a la acumulación de las deformaciones plásticas y a la ductilidad efectiva. Adicionalmente, los espectros de la derecha respecto a los de la izquierda, el incremento de la demanda fue aproximadamente $25 \%$ para $\mu_{u}=2$, y alrededor de $40 \%$ a $50 \%$ para $\mu_{u}=4$ y 6 , respectivamente. De manera que el aumento en la demanda de la resistencia dependió del valor de ductilidad última $\mu_{u}$. Finalmente, mientras mayor fue el periodo dominante del suelo y el valor de ductilidad, menor fue el periodo donde ocurrieron las mayores demandas de resistencia.

Las metodologías actuales para el diseño sísmico se basan en espectros de pseudo-aceleración simplistas, los cuales alcanzan una aceleración constante hasta cierto período y luego disminuye monotónicamente a medida que el mismo aumenta. Considerando que el efecto ISE alarga el periodo estructural y generalmente incrementa el coeficiente de amortiguamiento, se podría suponer una respuesta sísmica menor, por esta razón, los efectos de ISE se consideran despreciables. Sin embargo, acorde a los espectros de respuesta obtenidos, esto no es del todo correcto. Adicionalmente, dado que el cortante basal es la propiedad estructural a diseñar, $V_{b}=S_{a} W$ (donde $\mathrm{W}$ es el peso de la estructura), la figura 11 muestra una comparación entre un espectro que contempla los efectos de ISE y otro que no considera ISE ni daño acumulado (Sin Daño). Se observó que para periodos estructurales menores al periodo dominante del suelo, los efectos de ISE fueron perjudiciales, debido que fue donde ocurrieron las mayores demandas de resistencia. Mientras que para periodos estructurales mayores al periodo dominante del terreno, los efectos de ISE fueron benéficos, porque las demandas de resistencia obtenidas disminuyeron a medida que el periodo estructural se alejó del periodo del sitio.

Como se comentó, las deformaciones plásticas acumuladas incrementaron la demanda de resistencia. La figura 12 hace una comparación entre un espectro que se obtiene al considerar el efecto de ISE y daño acumulado simultáneamente (ISE+Daño), respecto aquel que no considera ninguno de los dos efectos (Sin Daño). Similar a la figura anterior, la ISE fue perjudicial para periodos estructurales menores al periodo del terreno y benéfico para el caso contario; sin embargo, al considerar el efecto de las demandas acumuladas, las demandas de resistencia se incrementaron de manera considerable, por lo tanto se obtuvo una fuerza de diseño mayor. Es claro que no contemplar los efectos de ISE y daño acumulado, puede derivar en diseños sísmicos que resulten del lado de la inseguridad. 

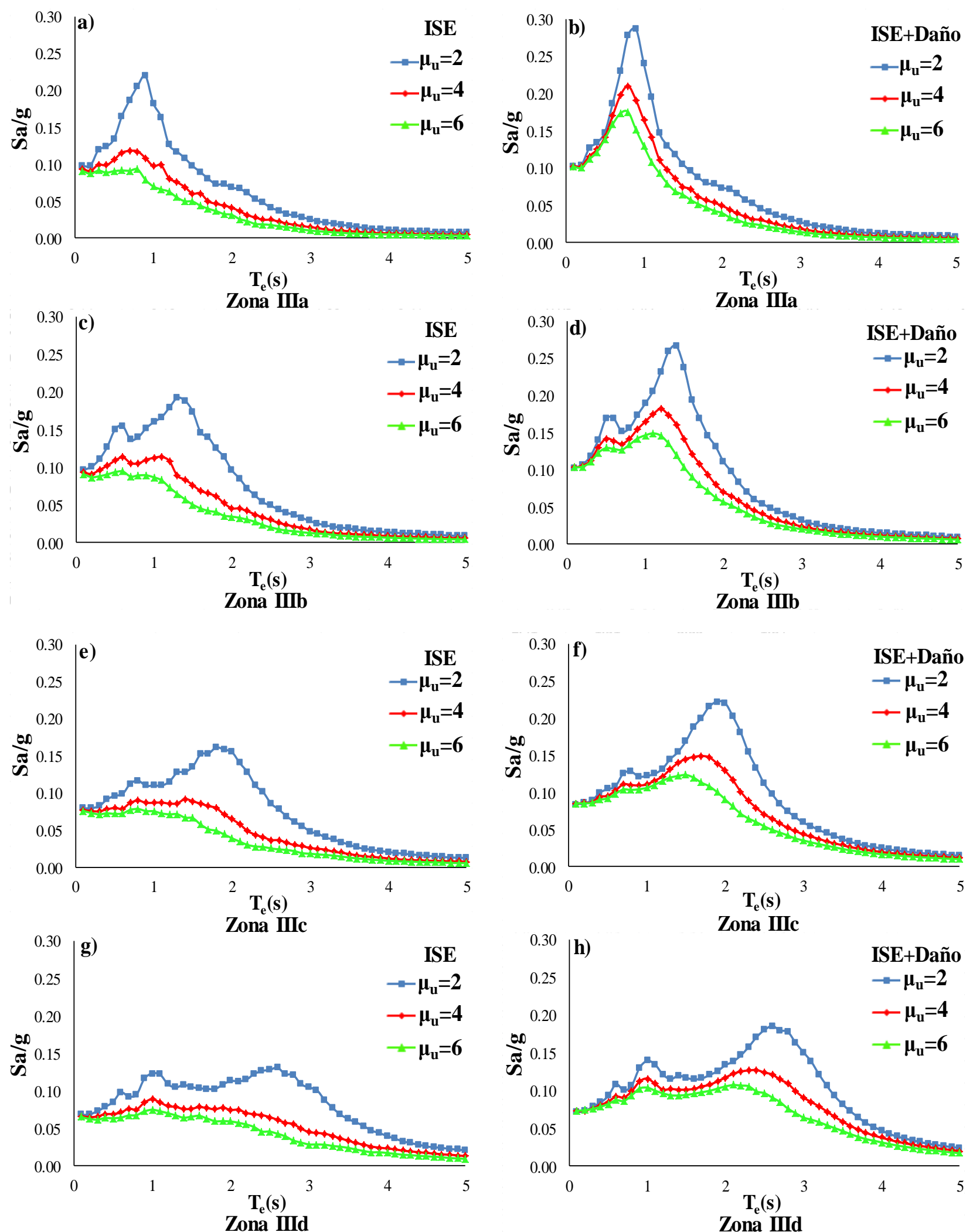

Figura 10. Espectros de respuesta promedio para sistemas elasto-plásticos para distintas zonas de la Ciudad de México; $\mu=2,4$ y 6 


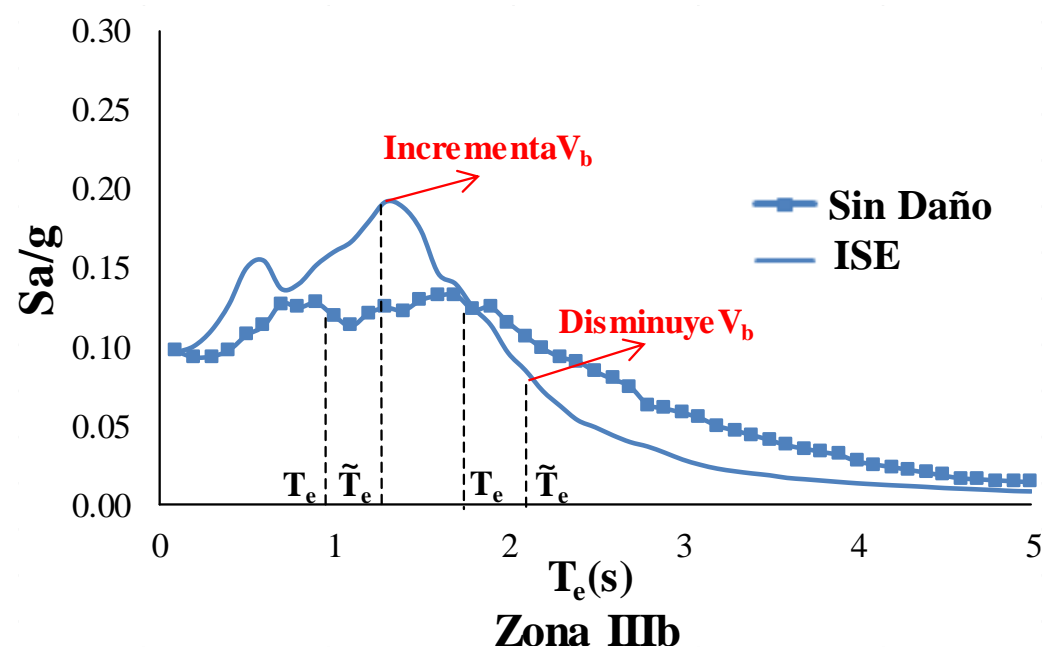

Figura 11. Comparación de espectros de respuesta para sistemas elasto-plásticos; $\mu_{\mathrm{u}}=2$, con y sin consideración de ISE (Zona IIIb)

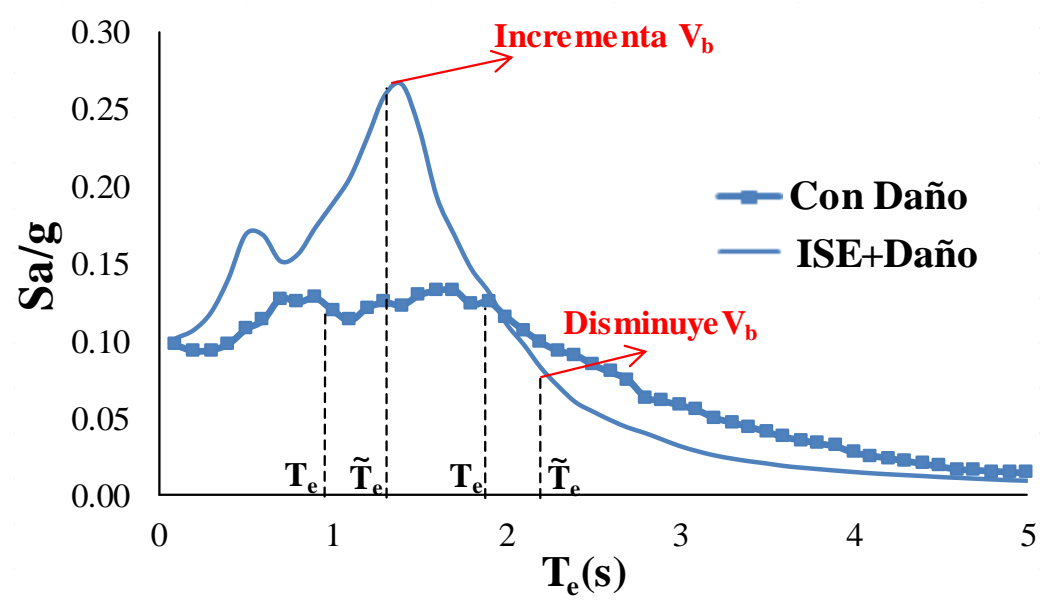

Zona IIIb

Figura 12. Comparación de espectros de respuesta para sistemas elasto-plásticos; $\mu_{\mathrm{u}}=2$, considerando ISE y daño acumulado (Zona IIIb)

\section{CONCLUSIONES}

Se determinó la respuesta inelástica de osciladores de un grado de libertad considerando interacción suelo-estructura (ISE) y las demandas acumuladas por deformación plástica. Para ello, se trazaron los espectros de respuesta elásticos e inelásticos, correspondientes a distintas zonas sísmicas de suelo blando de la ciudad de México. Para considerar los efectos de ISE, una estructura real de base-flexible puede ser remplazada por un oscilador equivalente no lineal con la misma resistencia y capacidad de disipación de energía que la estructura real, este último caracterizado por $\widetilde{T}_{e}, \widetilde{\zeta_{e}}$ y $\tilde{\mu}_{e}$, a pesar de ser un modelo simple, brinda una idea clara de los efectos de ISE; además, muchos conceptos de diseño en ingeniería sísmica han sido derivados a partir de un oscilador simple. Por otro lado, para la evaluación de la influencia del daño acumulado en el desempeño estructural, se introdujo el índice de daño de Park y Ang. 
Los resultados indican que al considerar la influencia de las demandas acumuladas en los espectros de respuesta, las ordenadas espectrales que se presentan son mayores, especialmente para estructuras que tengan un periodo estructural próximo al periodo del terreno, por lo que es deseable para fines de diseño tomar en cuenta dicho efecto. Es importante señalar que los resultados obtenidos dependen estrictamente de la capacidad que el índice de daño de Park y Ang tenga para evaluar apropiadamente el daño estructural, dado que este índice no considera la evolución del daño durante el movimiento en la respuesta, para la construcción de los espectros, antes de aplicar el movimiento, los modelos ya presentan el efecto del daño definido por el índice de Park y Ang. Sin embargo, el uso de diversos índices de daño, planteados a partir de simplificaciones y suposiciones muy diferentes, resulta en demandas de resistencia muy similares cuando se consideran las demandas acumuladas de deformación plástica.

Mientras que los efectos de ISE, pueden ser benéficos para estructuras que tengan periodos mayores al periodo del terreno y perjudiciales para el caso contrario. Los efectos de ISE dependen en gran medida de la rigidez relativa de la estructura y el suelo, y son depreciables para velocidades de onda de corte elevadas. Por otra parte, la solución del oscilador de reemplazo no lineal, depende particularmente de la capacidad que las expresiones utilizadas tengan para calcular adecuadamente el periodo $\mathrm{y}$ amortiguamiento efectivo. Cabe señalar que dado a lo complejo que puede resultar el cálculo de las funciones de impedancia para determinar el periodo y amortiguamiento efectivo, se usaron formulas aproximadas para estimar los coeficientes dinámicos de las funciones de impedancia. Sin embargo, de acuerdo a observaciones anteriores reportadas por otros autores, sugieren que las expresiones utilizadas para el cálculo del periodo y amortiguamiento efectivo son lo suficientemente adecuadas para propósitos ingenieriles, entre tanto, las fórmulas utilizadas, proporcionan datos útiles y apoyaran a interpretar adecuadamente los resultados obtenidos de análisis más sofisticados, esto de alguna manera aporta certeza a las tendencias observadas.

\section{AGRADECIMIENTOS}

Este trabajo se desarrolló con el apoyo económico brindado por El Consejo Nacional de Ciencia y Tecnología CONACYT, a través del Proyecto CB-2011-01-167419 y la Beca otorgada al primer autor. Se agradece el apoyo de la Universidad Autónoma de Sinaloa dentro del proyecto PROFAPI 2015, y a dos revisores anónimos por sus comentarios constructivos, recomendaciones y críticas que mejoraron la calidad del trabajo presentado.

\section{REFERENCIAS}

Arefi, M J (2008), "Effects of Soil-Structure Interaction on the Seismic Response of Existing R.C. Frame Buildings", Thesis for master degree in Earthquake Engineering \& Engineering Seismology, Istituto Universitario di Studi Superiori di Pavia.

Arroyo, D y M Ordaz (2007), "Hysteretic energy demands for SDOF systems subjected to narrow band earthquake ground motions. Applications to the lake bed zone of Mexico City", Journal of Earthquake Engineering, Vol. 11, pp. 147-165. DOI:10.1080/13632460601123131

Avilés, J y M Suárez (2002), "Effective periods and dampings of building-foundation systems including seismic wave effects", Earthquake Engineering and Structural Dynamics, Vol. 24(25), pp. 553-562. DOI:10.1016/S0141-0296(01)00121-3 
Avilés, J y L E Pérez-Rocha (2004), "Design concepts for yielding structures on flexible foundation", Earthquake Engineering and Structural Dynamics, pp. 443-454. DOI:10.1016/j.engstruct.2004.11.005

Avilés, J y L E Pérez-Rocha (2007), "Damage analysis of structures on elastic foundation”, Journal of Structural Engineering, ASCE, Vol. 133(10), pp.1453-1461. DOI:10.1061/(ASCE)07339445(2007)133:10(1453)

Bojórquez, E, J Bojórquez, A Terán-Gilmore y S E Ruiz Gómez (2009), “Consideración explicita del daño acumulado en el diseño sísmico de estructuras a través de factores de reducción de resistencia por ductilidad", Revista de ingeniería sísmica, SMIS, Vol. 80, pp. 31-62.

Bojórquez, E, A Reyes-Salazar, A Terán-Gilmore, SE Ruiz (2010). "Energy-based damage index for steel structures", Steel and Composite Structures, Vol. 10(4), pp. 331-348. DOI:10.12989/scs.2010.10.4.331

Bozorgnia, Y y V V Bertero (2001), "Improved shaking and damage parameters for post-earthquake applications", Proceedings of the SMIP01 Seminar on Utilization of Strong-Motion Data, Los Angeles, California.

Cosenza, E, G Manfredi y R Ramasco (1993), "The use of damage functionals in earthquake engineering: a comparison between different methods", Earthquake Engineering and Structural Dynamics, Vol. 22, pp. 855-868. DOI:10.1002/eqe.4290221003

Fajfar, P (1992), "Equivalent ductility factors, taking into account low-cycle fatigue", Earthquake Engineering and Structural Dynamics, Vol. 21, pp. 837-848. DOI:10.1002/eqe.4290211001

Fernández Sola, L R, J Avilés y D Muria (2012), “Distribución de elementos mecánicos en pilas sujetas a fuerzas en la cabeza y ante la incidencia de ondas sísmicas", Revista de Ingeniería Sísmica, SMIS, Vol. 87, pp. 1-23.

Gazetas, G (1991), "Formulas and charts for impedances of surface and embedded foundations", Journal of Geotechnical Engineering, Vol. 117, pp. 1363-1381. DOI:10.1061/(ASCE)07339410(1991)117:9(1363)

Jennings P C y J Bielak (1973), "Dynamics of building-soil interaction", Bulletin of the seismological society of America, Vol. 63(1), pp. 9-48.

Kausel, E, R V Whitman, J P Morray y F Elsabee (1978), "The spring method for embedded foundations", Nuclear Engineering and Design, Vol.48, pp. 377-92. DOI:10.1016/0029-5493(78)90085-7

Krawinkler, H y M Zohrei (1983), "Cumulative damage in steel structure subjected to earthquake ground motions", Computer and Structures, Vol. 16 pp. 531-541. DOI:10.1016/0045-7949(83)90193-1

Mylonakis, G y G Gazetas (2000), "Seismic soil-structure interaction: Beneficial or detrimental?", Journal Earthquake Engineering, Vol. 4(3), pp. 277-301. DOI:10.1080/13632460009350372

Park, Y J y A H Ang (1985), "Mechanistic seismic damage model for reinforced concrete", ASCE Journal of Structural Engineering, Vol. 111, No. ST4, pp. 740-757. DOI:10.1061/(ASCE)07339445(1985)111:4(722)

Reglamento de construcción de México (2004), "Normas Técnicas Complementarias para el Diseño por Sismo", Gobierno del Distrito Federal, Ciudad de México.

Rodríguez, M E (1997), "Una medida de la capacidad destructiva de terremotos", Revista de Ingeniería Sísmica, SMIS, Vol. 55, pp. 37-59. 
Rodríguez, M E y D Padilla (2008), “A damage index for the seismic analysis of reinforced concrete members", artículo aceptado para Journal of Earthquake Engineering. DOI:10.1080/13632460802597893

Stewart J P, S Kim, J Bielak, R Dobry y M S Power (2003), "Revisions to soil-structure interaction procedures in NEHRP design provisions", Earthquake Spectra, Vol. 19(3), pp. 677-696. DOI:10.1193/1.1596213

Tapia, E y A Tena (2014), “Code-Oriented methodology for the seismic design for regular steel moment resisting braced frames", Earthquake Spectra Journal, Vol. 3, No. 4, pp. 1683-1709. DOI:10.1193/032012EQS100M

Terán-Gilmore, A y J O Jirsa (2005), “A damage model for practical seismic design that accounts for low cycle fatigue”, Earthquake Spectra, Vol. 21(3), pp. 803-832. DOI:10.1193/1.1979500

Tolentino, D y S E Ruiz (2015), "Evaluación del factor de confianza considerando daño estructural sísmico en el tiempo", Revista de Ingeniería Sísmica, SMIS, Vol. 93, pp. 1-19.

Veletsos, A S y J W Meek (1974), "Dynamic behaviour of building-foundation systems", Earthquake Engineering and Structural Dynamics, Vol. 3, pp. 121-138. DOI:10.1002/eqe.4290030203

Villa-Velázquez, C I y S E Ruiz (2001), "Influencia de la intensidad en las características de los movimientos registrados en el valle de México", Informe elaborado para la Secretaría de Obras y Servicios del D.F., Instituto de Ingeniería, UNAM.

Wolf, J P (1988), Soil-structure interaction analysis in time domain, 1st Edition, Prentice-Hall, New Jersey. 
APÉNDICE A. REGISTROS SÍSMICOS UTILIZADOS (Villa-Velázquez y Ruiz, 2001)

Tabla A.1 Registros sísmicos de la zona IIIa

\begin{tabular}{ccccccc}
\hline & & & & \multicolumn{2}{c}{ Coordenadas de la Estación } & Ams \\
\cline { 5 - 6 } Registro & Fecha & Magnitud & Nombre de la Estación & Latitud & $\begin{array}{c}\text { Longitud } \\
\text { (cm/s }\end{array}$ \\
& & & & $\mathrm{N}$ & \\
\hline 1 & $89-04-25$ & 6.9 & Roma & 19.405 & 99.166 & 48.8 \\
2 & $89-04-25$ & 6.9 & Ibero & 19.345 & 99.13 & 25.5 \\
3 & $89-04-25$ & 6.9 & Jardines de Coyoacán & 19.313 & 99.127 & 34.4 \\
4 & $89-04-25$ & 6.9 & Lindavista & 19.493 & 99.128 & 30.3 \\
5 & $89-04-25$ & 6.9 & Miramontes & 19.283 & 99.125 & 36.4 \\
6 & $89-04-25$ & 6.9 & San Simón & 19.375 & 99.148 & 39.6 \\
7 & $89-04-25$ & 6.9 & Unidad Colonia IMSS & 19.434 & 99.165 & 52.2 \\
8 & $95-09-14$ & 7.3 & Culhuacán & 19.33 & 99.125 & 30.8 \\
9 & $95-09-14$ & 7.3 & Ibero & 19.345 & 99.13 & 31.2 \\
10 & $95-09-14$ & 7.3 & Jardines de Coyoacán & 19.313 & 99.127 & 30.2 \\
11 & $95-09-14$ & 7.3 & Miramontes & 19.283 & 99.125 & 27.6 \\
12 & $97-01-11$ & 6.9 & Culhuacán & 19.33 & 99.125 & 12.4 \\
13 & $97-01-11$ & 6.9 & Roma & 19.405 & 99.166 & 17.6 \\
14 & $97-01-11$ & 6.9 & Jardines de Coyoacán & 19.313 & 99.127 & 24.1 \\
15 & $97-01-11$ & 6.9 & Miramontes & 19.283 & 99.125 & 26.2 \\
16 & $97-01-11$ & 6.9 & San Simón & 19.375 & 99.148 & 17.5 \\
\hline
\end{tabular}

Tabla A.2 Registros sísmicos de la zona IIIb

\begin{tabular}{|c|c|c|c|c|c|c|}
\hline \multirow[b]{2}{*}{ Registro } & \multirow[b]{2}{*}{ Fecha } & \multirow[b]{2}{*}{ Magnitud } & \multirow[b]{2}{*}{ Nombre de la Estación } & \multicolumn{2}{|c|}{ Coordenadas de la Estación } & \multirow{2}{*}{$\begin{array}{c}\text { Ams } \\
\left(\mathrm{cm} / \mathrm{s}^{2}\right)\end{array}$} \\
\hline & & & & $\begin{array}{c}\text { Latitud } \\
\mathrm{N}\end{array}$ & $\begin{array}{c}\text { Longitud } \\
\text { W }\end{array}$ & \\
\hline 1 & 85-09-19 & 8.1 & SCT & 19.292 & 99.147 & 178 \\
\hline 2 & $85-09-21$ & 7.6 & Tlahuac deportivo & 19.293 & 99.035 & 48.7 \\
\hline 3 & $89-04-25$ & 6.9 & Alameda & 19.436 & 99.145 & 45 \\
\hline 4 & $89-04-25$ & 6.9 & Cibeles & 19.419 & 99.165 & 62.2 \\
\hline 5 & $89-04-25$ & 6.9 & CU Juárez & 19.41 & 99.157 & 49.1 \\
\hline 6 & $89-04-25$ & 6.9 & Garibaldi & 19.439 & 99.14 & 68 \\
\hline 7 & $89-04-25$ & 6.9 & SCT & 19.393 & 99.147 & 44.9 \\
\hline 8 & $89-04-25$ & 6.9 & Tlatelolco & 19.45 & 99.134 & 52.9 \\
\hline 9 & $89-04-25$ & 6.9 & Tlatelolco & 19.436 & 99.143 & 39.3 \\
\hline 10 & $95-09-14$ & 7.3 & Alameda & 19.436 & 99.145 & 39.1 \\
\hline 11 & $95-09-14$ & 7.3 & CU Juárez & 19.41 & 99.157 & 27.9 \\
\hline 12 & $95-09-14$ & 7.3 & Cto. Urbano Presidente Juárez & 19.41 & 99.157 & 27.7 \\
\hline 13 & $95-09-14$ & 7.3 & Córdoba & 19.422 & 99.159 & 43.3 \\
\hline 14 & $95-09-14$ & 7.3 & Edif. Jalapa & 19.423 & 99.159 & 29.6 \\
\hline 15 & $95-09-14$ & 7.3 & Garibaldi & 19.439 & 99.14 & 30.1 \\
\hline 16 & $95-09-14$ & 7.3 & Liconsa & 19.306 & 98.963 & 49.5 \\
\hline 17 & $95-09-14$ & 7.3 & Plutarco Elías Calles & 19.39 & 99.132 & 33.5 \\
\hline 18 & $95-09-14$ & 7.3 & Rodolfo Menéndez & 19.436 & 99.128 & 22.3 \\
\hline 19 & $95-09-14$ & 7.3 & Sector Popular & 19.366 & 99.119 & 34.3 \\
\hline 20 & $95-09-14$ & 7.3 & Tlatelolco & 19.45 & 99.134 & 27.5 \\
\hline
\end{tabular}


Tabla A.3 Registros sísmicos de la zona IIIc

\begin{tabular}{|c|c|c|c|c|c|c|}
\hline \multirow[b]{2}{*}{ Registro } & \multirow[b]{2}{*}{ Fecha } & \multirow[b]{2}{*}{ Magnitud } & \multirow[b]{2}{*}{ Nombre de la Estación } & \multicolumn{2}{|c|}{ Coordenadas de la Estación } & \multirow{2}{*}{$\begin{array}{c}\text { Ams } \\
\left(\mathrm{cm} / \mathrm{s}^{2}\right)\end{array}$} \\
\hline & & & & $\begin{array}{l}\text { Latitud } \\
\mathrm{N}\end{array}$ & $\begin{array}{l}\text { Longitud } \\
\text { W }\end{array}$ & \\
\hline 1 & $89-04-25$ & 6.9 & Buenos Aires & 19.41 & 99.145 & 61.9 \\
\hline 2 & $89-04-25$ & 6.9 & Candelaria & 19.426 & 99.118 & 48.7 \\
\hline 3 & $89-04-25$ & 6.9 & Prepa \# 7 La Viga & 19.419 & 99.126 & 46.7 \\
\hline 4 & $89-04-25$ & 6.9 & Jamaica & 19.405 & 99.125 & 36.4 \\
\hline 5 & $89-04-25$ & 6.9 & Villa del Mar & 19.381 & 99.125 & 44.2 \\
\hline 6 & $89-04-25$ & 6.9 & Xochipilli & 19.42 & 99.135 & 52.9 \\
\hline 7 & $95-09-14$ & 7.3 & Apatlaco & 19.381 & 99.107 & 51.6 \\
\hline 8 & $95-09-14$ & 7.3 & Candelaria & 19.426 & 99.118 & 31.4 \\
\hline 9 & $95-09-14$ & 7.3 & Central de abastos oficina & 19.372 & 99.096 & 36.9 \\
\hline 10 & $95-09-14$ & 7.3 & Jamaica & 19.405 & 99.125 & 30.8 \\
\hline 11 & $95-09-14$ & 7.3 & Meyehualco & 19.346 & 99.043 & 50.4 \\
\hline 12 & $95-10-09$ & 7.5 & Apatlaco & 19.381 & 99.107 & 15.8 \\
\hline 13 & $95-10-09$ & 7.5 & Buenos Aires & 19.41 & 99.145 & 22.7 \\
\hline 14 & $95-10-09$ & 7.5 & Candelaria & 19.426 & 99.118 & 20.9 \\
\hline 15 & $95-10-09$ & 7.5 & Jamaica & 19.405 & 99.125 & 13.3 \\
\hline 16 & $95-10-09$ & 7.5 & Xochipilli & 19.42 & 99.135 & 23.5 \\
\hline
\end{tabular}

Tabla A.4 Registros sísmicos de la zona IIId

\begin{tabular}{|c|c|c|c|c|c|c|}
\hline \multirow[b]{2}{*}{ Registro } & \multirow[b]{2}{*}{ Fecha } & \multirow[b]{2}{*}{ Magnitud } & \multirow[b]{2}{*}{ Nombre de la Estación } & \multicolumn{2}{|c|}{ Coordenadas de la Estación } & \multirow{2}{*}{$\begin{array}{c}\mathrm{Ams} \\
\left(\mathrm{cm} / \mathrm{s}^{2}\right)\end{array}$} \\
\hline & & & & $\begin{array}{c}\text { Latitud } \\
\mathrm{N}\end{array}$ & $\begin{array}{c}\text { Longitud } \\
\text { W }\end{array}$ & \\
\hline 1 & $89-04-25$ & 6.9 & Cetis 57 & 19.386 & 99.054 & 21 \\
\hline 2 & $89-04-25$ & 6.9 & Deportivo Moctezuma & 19.431 & 99.096 & 28.8 \\
\hline 3 & $89-04-25$ & 6.9 & Palacio de los deportes & 19.406 & 99.1 & 22.4 \\
\hline 4 & $95-09-14$ & 7.3 & Deportivo Moctezuma & 19.431 & 99.096 & 26.6 \\
\hline 5 & $95-09-14$ & 7.3 & Palacio de los deportes & 19.406 & 99.1 & 29.2 \\
\hline 6 & $95-10-09$ & 7.5 & Aragón & 19.481 & 99.076 & 17.5 \\
\hline 7 & $95-10-09$ & 7.5 & Autódromo & 19.392 & 99.087 & 15 \\
\hline 8 & $95-10-09$ & 7.5 & Deportivo Moctezuma & 19.431 & 99.096 & 17.5 \\
\hline 9 & $95-10-09$ & 7.5 & Hangares & 19.418 & 99.079 & 16.2 \\
\hline 10 & $95-10-09$ & 7.5 & Palacio de los deportes & 19.406 & 99.1 & 17.7 \\
\hline 11 & $95-10-09$ & 7.5 & Est. \# 7 Tlacotal & 19.397 & 99.105 & 12.1 \\
\hline 12 & $95-10-09$ & 7.5 & Est. \# 8 Zaragoza & 19.419 & 99.088 & 16.9 \\
\hline 13 & $97-01-11$ & 6.9 & Autódromo & 19.392 & 99.087 & 22.3 \\
\hline 14 & $97-01-11$ & 6.9 & Cetis 57 & 19.386 & 99.054 & 14 \\
\hline 15 & $97-01-11$ & 6.9 & Palacio de los deportes & 19.406 & 99.1 & 20.8 \\
\hline 16 & $97-01-11$ & 6.9 & Est. \# 8 Zaragoza & 19.419 & 99.088 & 25.6 \\
\hline
\end{tabular}

